\title{
Searching for the happy medium in the therapeutic approach to childhood sleep disordered breathing
}

\author{
Francisco Campos-Rodriguez ${ }^{1}$ and Miguel A. Martínez-García ${ }^{2,3}$
}

\begin{abstract}
Affiliations: ${ }^{1}$ Respiratory Dept, Hospital Universitario de Valme, Seville, Spain. ${ }^{2}$ Respiratory Dept, Hospital Universitario y Politecnico La Fe, Valencia, Spain. ${ }^{3}$ CIBERES, CIBER de enfermedades respiratorias, Madrid, Spain.
\end{abstract}

Correspondence: F. Campos-Rodriguez, Respiratory Dept, Hospital Universitario de Valme, Ctra Cadiz S/N, 41014, Sevilla, Spain. E-mail: fracamrodagmail.com

$\circ$ @ERSpublications

Is the optimum approach to childhood sleep disordered breathing prompt surgical intervention or watchful waiting? http://ow.ly/Xypal

The natural history of a disease is the course it takes in an individual, without medical intervention, from its pathological onset until its eventual resolution or death. Knowledge of this natural history is essential for understanding the disease and designing the most effective strategies for its prevention and control.

Sleep disordered breathing (SDB) is a relatively novel disease and we have little knowledge about its onset, causes and course. At present, we are limited to several risk factors that cannot fully explain the different phenotypes and features observed in specific populations such as middle-aged men, women, the elderly and children. As with other chronic disorders, it is difficult to identify the precise onset of SDB and trace its natural history. Moreover, the impairment of health status and cardiovascular risks associated with this disorder means that there are ethical implications in withholding treatment to observe its evolution over time. In this respect, children may offer a unique possibility for better tracing the onset and course of the disease, given the fewer coexisting comorbidities and chronic diseases in this population.

The paper by BIXLer et al. [1] in the current issue of European Respiratory Journal sheds some light on the natural history of SDB in the transition from childhood to adolescence, by reassessing a large cohort of children 8 years after baseline evaluation. This study provides two main findings: 1) all children with severe SDB (apnoea-hypopnoea index (AHI) $>5$ episodes per hour) and most with moderate SDB (defined in this study as an AHI of 2-5 episodes per hour) experienced either partial or complete remission in the AHI; and 2) risk factors for SDB in adolescence were similar to those found in adulthood but were very different from those observed in childhood.

Spontaneous remission of SDB may change the therapeutic approach to this disorder in children, thereby opening up the question of whether to apply active treatment or watchful waiting as the first-line treatment. Current guidelines recommend active treatment for those children with an AHI $>5$ episodes per hour or an AHI of 1-5 episodes per hour and the presence of cardiovascular morbidity, symptomatic disease or factors predicting SDB persistence [2]. These recommendations are based on strong evidence demonstrating that SDB may impair quality of life and increase blood pressure, hyperactivity symptoms, cognitive deficits, academic difficulties and behavioural disorders [3-6]. The preferred treatment in children is adenotonsillectomy [2], which in the study by BIXLER et al. [1] showed a trend, albeit nonsignificant, towards an increased incidence of SDB in adolescence, a finding also reported in other studies [7]. Therefore, the key question would be to choose between an early treatment, assuming the risks of surgery or even the possibility that surgery itself may pose an additional risk for the development of

Received: Jan 112016 | Accepted: Jan 122016

Conflict of interest: None declared.

Copyright CERS 2016 
SDB at an older age, and waiting in the hope that, in most cases, the disease will remit with growth. Although the choice of waiting, which is supported by the results of this study, seems prudent and may save useless surgery, it does raise important concerns.

First, although this study concurs with others that have observed remission rates in children of between $42 \%$ and $90 \%$ [7-9], with most adolescent cases being of new onset, there are other researchers who have not confirmed this trend of spontaneous resolution. In a recent study involving children with mild SDB, one third of the sample progressed to a more severe state after a follow-up of 2 years [10]. It is likely that this discrepancy can be attributed to different characteristics of the study population and a small sample size, but these findings nevertheless warn that a spontaneous resolution of SDB cannot be taken for granted in all children, and therefore predictors for progression should be identified.

Secondly, it is unclear how long we should wait until more aggressive management is introduced. The time span of 8 years in the paper of BIXLER et al. [1] seems too long, considering the potential adverse health outcomes and the risk of progression of SDB. It is necessary to define clear-cut follow-up appointments to reassess whether SDB remits or not, not only from a polysomnographic, but also from a clinical, point of view. The cost-effectiveness of repeated clinical appointments and sleep tests should be weighed against early surgical treatment, to decide which of these two strategies is preferable in this population. In this respect, it seems that watchful waiting periods as short as 7 months may achieve remission rates of $42-46 \%[8,9]$.

A third concern is whether the sleep study metric, i.e. the AHI, really reflects the overall burden of SDB in children. Long-term, permanent consequences, such as cognitive impairment, growth failure and cardiovascular disease, may occur in children with high AHI, but also in those with only habitual snoring or low AHI $[11,12]$. Thus, if even mild disease may provoke long-term sequelae, it would be risky to withhold treatment on the sole basis of a foreseeable reduction in the AHI. Along these lines, a large randomised controlled trials compared early adenotonsillectomy and watchful waiting in children of 5 to 9 years of age [8]. After a follow-up of 7 months, no differences in attention or executive function were found between the strategies but early surgery did reduce symptoms and improve behaviour and quality-of-life outcomes. Another study observed only a $15 \%$ improvement in SDB symptoms despite a $42 \%$ spontaneous reduction in AHI after 7 months of follow-up [9]. In this study, only $12 \%$ of the children had both polysomnographic and symptomatic resolution under watchful waiting. Unfortunately, in the present study by BIXLER et al. [1], data regarding changes in blood pressure, physical examination and psychometric assessment were not provided. This information would have been very useful to confirm whether the observed AHI decrease was associated with an improvement in symptoms and, more importantly, an absence of long-term adverse outcomes, or whether, on the contrary, some children had experienced non-reversible consequences in spite of the reduction in AHI metrics. It is probable that, given the different evolutions of the disease, which may correspond to different phenotypes, the aid of personalised medicine would provide an invaluable tool for identifying which children are not exposed to severe consequences and can be managed with watchful waiting and which ones should be treated early to prevent long-lasting sequelae [13].

The other interesting finding of this paper is the identification of risk factors associated with the new onset of SDB in adolescence. Remarkably, primary snoring and mild disease in childhood were poor predictors, whereas older age, obesity and male sex, the usual risk factors in adults, were independently associated with SDB in adolescence, as previously reported in recent studies [7, 14]. This underscores the differences in pathophysiology across the growth stages and shows how adolescent SDB more closely resembles the sleep disorder found in adulthood than that found in childhood. It is likely that the changing size of the adenotonsillar tissue [15], combined with other risk factors such as smaller lung volumes or central ventilatory instability, lead to dynamic changes in the collapsibility of the upper airway that would play a key role in the development of SDB in children. In adolescence, once the upper airway is more stable and pubertal alterations occur in hormones and body mass index, these factors are replaced by others more similar to those usually found in adults. These distinct predisposing factors help to explain the trend towards remission in the transition from childhood to adolescence, and suggest that SDB, as we usually recognise it in adulthood, is likely to start during adolescence. Whether this is enough to consider childhood and adolescent/adult SDB as different disorders is uncertain. Anyway, the real challenge is to define and accomplish screening strategies tailored to the early detection of the onset of SDB in adolescents that may help us avoid its progression and its consequences throughout adulthood.

In summary, this paper suggests that childhood SDB usually remits, at least partly because of the change in predisposing factors during growth. However, it is uncertain whether this polysomnographic remission is accompanied by symptom resolution and the avoidance of long-term health consequences, or whether watchful waiting is a better therapeutic strategy than active treatment. We are faced with the challenge of 
identifying which children merit prompt surgical treatment to prevent the occurrence of complications and in which ones it is reasonable to wait in the hope that SDB will remit as the child grows up. In these latter cases, the time span between clinical assessments and the cost-benefits of this strategy should be clarified. Personalised medicine will undoubtedly help us in this task.

\section{References}

1 Bixler EO, Fernandez-Mendoza J, Liao D, et al. Natural history of sleep disordered breathing in prepubertal children transitioning to adolescence. Eur Respir J 2016; 47: 1402-1409.

2 Kaditis AG, Alonso Alvarez ML, Boudewyns A, et al. Obstructive sleep disordered breathing in 2- to 18-year-old children: diagnosis and management. Eur Respir J 2016; 47: 69-94.

3 Amin RS, Carroll JL, Jeffries JL, et al. Twenty-four-hour ambulatory blood pressure in children with sleep-disordered breathing. Am J Respir Crit Care Med 2004; 169: 950-956.

4 Baldassari CM, Mitchell RB, Schubert C, et al. Pediatric obstructive sleep apnea and quality of life: a meta-analysis. Otolaryngol Head Neck Surg 2008; 138: 265-273.

5 Sedky K, Bennett DS, Carvalho KS. Attention deficit hyperactivity disorder and sleep disordered breathing in pediatric populations: a meta-analysis. Sleep Med Rev 2014; 18: 349-356.

6 Perfect MM, Archbold K, Goodwin JL, et al. Risk of behavioral and adaptive functioning difficulties in youth with previous and current sleep disordered breathing. Sleep 2013; 36: 517-525B.

7 Spilsbury JC, Storfer-Isser A, Rosen CL, et al. Remission and incidence of obstructive sleep apnea from middle childhood to late adolescence. Sleep 2015; 38: 23-29.

8 Marcus CL, Moore RH, Rosen CL, et al. A randomized trial of adenotonsillectomy for childhood sleep apnea. $N$ Engl J Med 2013; 368: 2366-2376.

9 Chervin RD, Ellenberg SS, Hou X, et al. Prognosis for spontaneous resolution of OSA in children. Chest 2015; 148: 1204-1213

10 Li AM, Au CT, Ng SK, et al. Natural history and predictors for progression of mild childhood obstructive sleep apnoea. Thorax 2010; 65: 27-31.

11 O'Brien LM, Mervis CB, Holbrook CR, et al. Neurobehavioral implications of habitual snoring in children. Pediatrics 2004; 114: 44-49.

12 Sans Capdevila O, Crabtree VM, Kheirandish-Gozal L, et al. Increased morning brain natriuretic peptide levels in children with nocturnal enuresis and sleep-disordered breathing: a community-based study. Pediatrics 2008; 121 : e1208-e1214.

13 Tan H-L, Kheirandish-Gozal L, Gozal D. The promise of translational and personalised approaches for paediatric obstructive sleep apnoea: an "Omics" perspective. Thorax 2014; 69: 474-480.

14 Alonso-Álvarez ML, Terán-Santos J, Navazo-Egüia AI, et al. Treatment outcomes of obstructive sleep apnoea in obese community-dwelling children: the NANOS study. Eur Respir J 2015; 46: 717-727.

15 Akcay A, Kara CO, Dagdeviren E, et al. Variation in tonsil size in 4- to 17-year-old schoolchildren. J Otolaryngol 2006; 35: 270-274. 